\title{
Continuous Cohomology and a Conjecture of Serre's
}

\author{
W. Casselman * (Vancouver) and D. Wigner (Ann Arbor)
}

0 . Let $G$ be the group of $\mathbb{Q}_{p}$-rational points on a connected semisimple group defined over $\mathbb{Q}_{p}, \mathscr{G}$ its Lie algebra, $H^{*}\left(G, \mathbb{Q}_{p}\right)$ the continuous cohomology of $G$ with coefficients in $\mathbb{Q}_{p}$. When $G$ is compact, a result of Lazard's ([7], Chapter V, Theorem 2.4.10) and an argument about Zariski-closure (see §3) imply that $H^{*}\left(G, \mathbb{Q}_{p}\right) \cong H^{*}\left(\mathscr{G}, \mathbb{Q}_{p}\right)$. The original motivation for most of the results in this paper was the question asked by Serre ([11], p. 119): Does Lazard's result hold for more general $G$ ? We show this to be so (Theorem 1 in $\S 3$ ).

We include a largely self-contained exposition of continuous cohomology theory for locally compact groups. Our main result here is a form of Shapiro's Lemma (Propositions 3 and 4 in $\$ 1$ ). We also include a discussion of the Hochschild-Serre spectral sequence (in $\$ 2$ ). We have drawn largely on a paper of Hochschild and Mostow [4] which treats the case of $G$-modules which are real vector spaces, but our emphasis is quite different.

In many cases our cohomology agrees with that constructed by Calvin Moore (described in [10]), and a number of our results are implied by results of his.

In $\S 3$ we apply Shapiro's Lemma and the Bruhat-Tits building to prove Serre's conjecture. In $\$ 4$ we deal with the cohomology of $p$-adic groups with coefficients in real vector spaces, including some remarks about the cohomology of smooth representations over more general fields. In $\S 5$ we answer a question of Serre's about the analytic cohomology of a $p$-adic semi-simple group.

We wish to thank A. Bore! for several valuable suggestions; P. Cartier for remarks concerning $\S 2$; and R. Bott for explaining to one of us a long time ago how a form of Shapiro's Lemma offered a simple proof of Theorem 6.1 in [4]. (It was this explanation which ultimately suggested the proof of our Theorem 1.) Finally, we wish to thank the referee for the great deal of patience involved in reading and correcting several versions of this paper, and more particularly for suggesting the proof of Hochschild-Serre that we give.

1. Let $G$ be a locally compact topological group. Define a $G$-space to be a topological Hausdorff space $A$ together with a jointly continuous

\footnotetext{
* The first author has been supported by grants from the National Research Council of Canada and the National Science Foundation while preparing this.
}

14 Invertiones math., Vol. 25 
associative $G$-action: $G \times A \rightarrow A$. Define a $G$-module to be a $G$-space which is a topological abelian group such that the operations by elements of $G$ are group automorphisms. (We shall always write the product on $A$ additively.) If $A$ is a $G$-space, let $A^{G}$ be the (closed) set of points fixed by $G$.

If $A$ and $B$ are $G$-spaces define $\mathscr{H} r m(A, B)$ to be the set of all continuous maps from $A$ to $B$. If one gives it the compact-open topology and defines a $G$-action by the formula $(g f)(a)=g \cdot f\left(g^{-1} a\right)$, it becomes a $G$-space, and a $G$-module if $B$ is one. If $A$ and $B$ are $G$-modules, define $\operatorname{Hom}(A, B)$ to be the set of all continuous homomorphisms from $A$ to $B$. This similarly becomes a $G$-module.

If $H$ is any closed subgroup of $G$ and $A$ an $H$-space, define Ind $(A \mid H, G)$, or merely $I_{A}$ for short when confusion is unlikely, to be the space of all continuous maps $f: G \rightarrow A$ such that $f(g h)=h^{-1} \cdot f(g)$ for all $h \in H, g \in G$. If one gives it the compact-open topology and defines a $G$-action by the formula $(g f)(x)=f\left(g^{-1} x\right)$, one has a $G$-space, that induced by $A$ from $H$. Another way of describing $I_{A}$ is as $\mathscr{H}_{o m_{H}}(G, A)$, where one lets $H$ act on $G$ on the right: $g \mapsto g h^{-1}$. From this one sees immediately that $I_{A}$ is Hausdorff, since $\mathscr{H}_{0 m}(G, A)$ is and $I_{A}$ is closed in it. Define the canonical map $\theta: I_{A} \rightarrow A$ by the formula $\theta f=f(1)$. This is an $H$-morphism.

Lemma 1. If $A$ is already a G-module, then there exists a $G$-isomorphism of $\operatorname{Ind}(A \mid H, G)$ with $\mathscr{H}$ om $(G / H, A)$.

Proof. Associate to $f \in I_{A}$ the function $\phi: G / H \rightarrow A$ such that $\phi(g)=$ $g f(g)$. Associate to $\phi \in \mathscr{H}$ om $(G / H, A)$ the function $f$ such that $f(g)=$ $g^{-1} \phi(g)$.

Lemma 2 (Frobenius reciprocity). If $A$ is a $G$-space and $B$ an $H$-space, then the map $\theta$ induces an isomorphism of $\mathscr{H}_{0 m_{G}}\left(A, I_{B}\right)$ with $\mathscr{H}_{a m_{H}}(A, B)$. Similarly for Hom, if $A$ and $B$ are modules.

Proof. Since $G$ is locally compact, one has $\mathscr{H}$ om $(A, \mathscr{H}$ om $(G, B)) \cong$ $\mathscr{H}$ om $(A \times G, B)$ as topological spaces ([1], $\S 4$, Corollary 2 to Theorem 3 , pp. 46-47). If one considers the $G$-fixed elements of each side, one gets $\mathscr{H a m}_{G}\left(A, \mathscr{H}_{a m}(G, B)\right)$ isomorphic to the space of all maps $f: A \times G \rightarrow B$ such that $f(g a, g)=f(a, 1)$ for all $g \in G, a \in A$. The restriction of $f$ to $A \times\{1\}$ induces in turn an isomorphism of this space with $\mathscr{H}$ om $(A, B)$. If one considers the $\boldsymbol{H}$-fixed elements, the proposition follows. (Note: One may check that the isomorphism is actually the one induced by $\theta$. The technical problem here is to show that the isomorphism is a topological one.)

Lemma 3. The map $\theta$ induces an isomorphism of $I_{A}^{G}$ with $A^{H}$.

Proof. Trivial to prove directly, but it is also a special case of Lemma 2, with $A$ there a trivial $G$-space of one element. 
Lemma 4. If $A$ is any locally compact $G$-space and $B$ an $H$-space, then $I_{\mathscr{H} o m(A, B)} \cong \mathscr{H}$ om $\left(A, I_{B}\right)$ as $G$-spaces. Similarly for Hom, if $A$ and $B$ are modules.

Proof. By Corollary 2, p. 47 of [1] again, $\mathscr{H}$ om $(A, \mathscr{H}$ m $(G, B)) \cong$ $\mathscr{H}_{0 m}(A \times G, B) \cong \mathscr{H}_{0 m}\left(G, \mathscr{H}_{0 m}(A, B)\right)$. Apply the interpretation of $I_{C}$ as $\mathscr{H}_{0 m_{H}}(G, C)$ for the $H$-spaces $C=B$ and $C=\mathscr{H} o m(A, B)$.

If $A$ and $B$ are $G$-modules, a strong $G$-injection of $A$ into $B$ is a $G$ morphism for which a continuous left inverse (not necessarily a homomorphism) exists. A strong $G$-morphism $f: A \rightarrow B$ is a $G$-morphism for which Ker $f \rightarrow A$ and Coim $f \rightarrow B$ are strong $G$-injections. In general, the composition of strong morphisms is not strong.

A $G$-module $A$ is said to be continuously injective if for every strong $G$-injection $U \rightarrow V$ and every $G$-morphism $f: U \rightarrow A$ there exists a $G$ extension of $f$ to $V$. Every topological abelian group is continuously injective with respect to the trivial group. If $A$ is a continuously injective $H$-module, then $\operatorname{Ind}(A \mid H, G)$ is a continuously injective $G$-module (this from Lemma 2).

If $A$ is a $G$-module, define $C^{n}(G, A)$, the space of continuous $n$-cochains on $G$ with values in $A$, to be $\mathscr{H}_{o m}\left(G^{n+1}, A\right)$. The left regular representation of $G$ on each factor defines $G^{n+1}$, hence $C^{n}(G, A)$, as a $G$-module. By Lemma $1, C^{0}(G, A) \cong \operatorname{Ind}(A \mid 1, G)$, and further one may see easily that $C^{n+1}(G, A) \cong C^{0}\left(G, C^{n}(G, A)\right)$. Thus each $C^{n}(G, A)$ is continuously injective. Every $G$-module $A$ has a canonical strong $G$-injection into $C^{0}(G, A)$, and if one applies this process in turn to the quotient, etc., one obtains the canonical strong resolution of $A$ by the complex $C^{*}(G, A)$ with the differential $d_{n}: C^{n} \rightarrow C^{n+1}$ defined by the familiar formula

$$
\left(d_{n} f\right)\left(g_{0}, g_{1}, \ldots, g_{n+1}\right)=\sum_{m}(-1)^{m} f\left(g_{0}, \ldots, \hat{g}_{m}, \ldots, g_{n+1}\right) \text {. }
$$

Define $H^{n}(G, A)$, the $n$-th continuous cohomology group of $G$ with coefficients in $A$, to be the $n$-th cohomology group of the complex $C^{*}(G, A)^{G}$. Of course $H^{0}(G, A) \cong A^{G}$.

If $0 \rightarrow A \rightarrow B \rightarrow C \rightarrow 0$ is a short exact sequence of strong morphisms, so is the induced sequence $0 \rightarrow C^{n}(G, A) \rightarrow C^{n}(G, B) \rightarrow C^{n}(G, C) \rightarrow 0$. One has therefore an associated long exact sequence of cohomology.

A $G$-module $A$ is called acyclic if $H^{n}(G, A)=0$ for $n>0$.

The groups $H^{n}(G, A)$ inherit a topology from that of the cochain spaces, which we shall call the canonical topology. We shall call these groups strongly Hausdorff if the injection of every

$$
B^{n}(G, A) \cong C^{n-1}(G, A) / Z^{n-1}(G, A)
$$

into $Z^{n}(G, A)$ is a strong injection (where $Z^{n}$ are the cocycles in $C^{n}$ ). This implies at least that the canonical topology on $H^{*}(G, A)$ is Hausdorff. 
(The terminology is faulty, since whether the groups are strongly Hausdorff or not is not an intrinsic property, but depends on their definition. This will cause no trouble.)

More generally, if $C^{*}$ is any complex of $G$-modules, one may similarly define its cohomology to be strongly Hausdorff if the injections of coboundaries $B^{*}$ into cocycles $Z^{*}$ are strong.

If $A^{*}$ is any strong resolution of $A$ by continuously injective $G$ modules, then the groups $H^{n}(G, A)$ are the cohomology groups of $\left(A^{*}\right)^{G}$. The topology on $H^{*}(G, A)$, and whether or not these groups are strongly Hausdorff, is independent of the resolution.

The following result implies that $H^{*}(G, A)$ may be computed from any acyclic resolution of $A$.

Proposition 1. If

$$
0 \rightarrow A \rightarrow A^{0} \rightarrow A^{1} \rightarrow A^{2} \rightarrow \cdots
$$

is a strong resolution of $A$ by G-modules, then there exists a spectral sequence converging to $H^{*}(G, A)$ with $E_{2}^{p . q} \cong H^{q}\left(H^{p}\left(G, A^{*}\right)\right)$.

Proof. One has this resolution of $A$ and the complex $A^{*}$ :

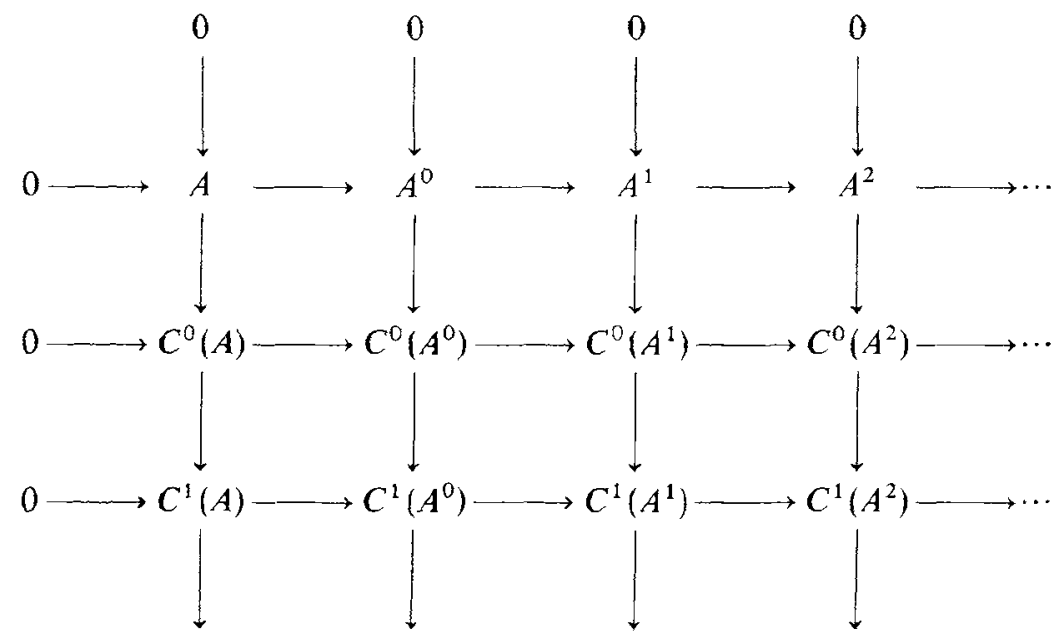

where we have written $C^{n}(*)$ instead of $C^{n}(G, *)$. Apply the functor $(*)^{G}$ to the complex $C^{p}\left(A^{q}\right)$, and then apply the usual spectral sequence arguments to the resulting double complex. One obtains by one filtration $H^{*}(G, A)$ (because the horizontal cohomology is trivial) and by the other the spectral sequence desired. 
Proposition 2. Let

$$
0 \rightarrow A^{0} \rightarrow A^{1} \rightarrow A^{2} \rightarrow \cdots
$$

be any complex of acyclic G-modules and G-morphisms whose cohomology is strongly Hausdorff. Then there exists a spectral sequence converging to the cohomology of the complex

$$
0 \rightarrow\left(A^{0}\right)^{G} \rightarrow\left(A^{1}\right)^{G} \rightarrow\left(A^{2}\right)^{G} \rightarrow \cdots
$$

with $E_{2}^{p, q} \cong H^{p}\left(G, H^{q}\left(A^{*}\right)\right)$.

Proof. Resolve $A^{*}$ by $C^{*}\left(A^{*}\right)$, apply the function $(*)^{G}$, and compute the two spectral sequences one has at hand. This time the vertical cohomology is trivial. The assumption on the topology of $H^{*}\left(A^{*}\right)$ is used to identify $C^{p}\left(G, H^{*}\right)$ with the quotient $C^{p}\left(G, Z^{*}\right) / C^{p}\left(G, B^{*}\right)$.

Proposition 3 (Shapiro's Lemma). Let $G$ be a locally compact group, $H$ a closed subgroup of $G$. Let $A$ be an $H$-module, and suppose that each $C^{n}(G, A)$ is acyclic as an $H$-module. Then the map from $H^{*}\left(G, I_{A}\right)$ to $H^{*}(H, A)$ induced by $\theta$ and the inclusion of $H$ in $G$ is an isomorphism.

Proof. Consider the standard resolution of $I_{A}$ by the cochain modules:

$$
0 \rightarrow I_{A} \rightarrow C^{0}\left(G, I_{A}\right) \rightarrow C^{1}\left(G, I_{A}\right) \rightarrow \cdots
$$

The cohomology $H^{*}\left(G, I_{A}\right)$ is that of the compiex

$$
0 \rightarrow C^{0}\left(G, I_{A}\right)^{G} \rightarrow C^{1}\left(G, I_{A}\right)^{G} \rightarrow \cdots
$$

By Lemma $4, C^{n}\left(G, I_{A}\right)=\mathscr{H}_{o m}\left(G^{n+1}, I_{A}\right) \cong I_{\mathscr{H}_{o m}\left(G^{n+1}, A\right)}=I_{C^{n}(G, A)}$, and by Lemma $3, C^{n}\left(G, I_{A}\right)^{G} \cong C^{n}(G, A)^{H}$. This isomorphism is natural, so that the cohomology $H^{*}\left(G, I_{A}\right)$ is that of the complex $C^{*}(G, A)^{H}$. By assumption, the complex $C^{*}(G, A)$ is a resolution of $A$ by acyclic $H$-modules, so that by the remark preceding Proposition 2, the cohomology of $C^{*}(G, A)^{H}$ is $H^{*}(H, A)$.

This proof is only a more technical version of the proof of Shapiro's Lemma for discrete groups (see [12]).

Proposition 4. (a) Suppose that the canonical projection $\pi: G \rightarrow H \backslash G$ has a continuous section. Then any continuously injective G-module is continuously injective as an $\mathrm{H}$-module.

(b) Suppose that $H \backslash G$ is paracompact, the projection $\pi: G \rightarrow H \backslash G$ has local continuous sections, and $A$ is a vector space over $\mathbb{R}$. Then each $C^{n}(G, A)$ is a continuously injective $H$-module.

Proof of (a). Every $G$-module $A$ has a canonical strong injection into $\mathscr{H}$ try $(G, A)(A$ injects as the constant functions, and $f \mapsto f(1)$ gives the splitting necessary for this to be a strong injection). If $A$ is continuously injective, $A$ will be a summand of $\mathscr{H}_{0 m}(G, A)$, so that it suffices to prove 
that $\mathscr{H} o m(G, A)$ is a continuously injective $H$-module, for every $G$ module $A$.

If $s$ is a continuous section of $\pi$, then the map from $G$ to $H \times H \backslash G$ taking $g$ to $\left(g \cdot(s(\pi(g)))^{-1}, \pi(g)\right)$ is an $H$-isomorphism (with $H$ acting on the left on itself, and trivially on $H \backslash G$ ). Thus

$$
\mathscr{H}_{o m}(G, A) \cong \mathscr{H}_{o m}(H \times H \backslash G, A) \cong \mathscr{H} \text { om }\left(H, \mathscr{H}_{o m}(H \backslash G, A)\right) \text {, }
$$

and we have remarked earlier that this last is a continuously injective $H$-module.

\section{Proof of (b). This is Lemma 3.4 in [4].}

Remarks. (1) When $G$ is metrizable and $H \backslash G$ is totally disconnected i.e., has as basis for the neighborhoods of some, hence any, point a (countable) set of open and closed subsets - then a continuous crosssection of $\pi: G \rightarrow H \backslash G$ always exists (see [9], Corollary 2). This is so in particular when $G$ is an analytic group over some non-archimedean locally compact field. In this case, one knows even that an analytic crosssection exists. Incidentally, if $G$ is $\sigma$-compact, totally disconnected, and $A$ a complete metric $G$-module, one knows ([13], Theorem 1) that the continuous cohomology of $G$ with coefficients in $A$ agrees with that of Calvin Moore in [10].

(2) Combining Propositions 1 and 2 we obtain: If the projection $\pi: G \rightarrow H \backslash G$ has a continuous cross-section, then for each $H$-module $A$ the map from $H^{*}\left(G, I_{A}\right)$ to $H^{*}(H, A)$ induced by $\theta$ and the inclusion of $H$ in $G$ is an isomorphism. We present here a second proof of this, because it has applications to cohomology theories other than that of continuous cohomology. This second proof begins as the first does, establishing that $H^{*}\left(G, I_{A}\right)$ is the cohomology of the complex $C^{*}(G, A)^{H}$. But from this point: let $i_{c}$ be the map from $C^{n}(G, A)^{H}$ to $C^{n}(H, A)^{H}$ induced by $i: H \rightarrow G$. Let $s$ be a cross-section of $\pi$. Then one obtains a morphism $\sigma$ of $H$-spaces ( $H$ acting on the left): $G \rightarrow H$ by the formula $\sigma(g)=g \cdot(s(\pi(g)))^{-1}$. This induces a chain map $\sigma_{c}: C^{n}(H, A)^{H} \rightarrow C^{n}(G, A)^{H}$. The composite $i_{c} \circ \sigma_{c}$ is the identity on $C^{n}(H, A)^{H}$. One also has

$$
\sigma_{c} \circ i_{c}(f)\left(g_{0}, \ldots, g_{n}\right)=f\left(\sigma\left(g_{0}\right), \sigma\left(g_{1}\right), \ldots, \sigma\left(g_{n}\right)\right) .
$$

Now define the map $\kappa: C^{n+1}(G, A) \rightarrow C^{n}(G, A)$ by the formula

$$
\kappa f\left(g_{0}, \ldots, g_{n}\right)=\sum_{j=0}^{n}\left(-1^{j} f\left(g_{0}, \ldots, g_{j}, \sigma\left(g_{j}\right), \ldots, \sigma\left(g_{n}\right)\right) .\right.
$$

It is straightforward to verify that $\kappa$ is a chain homotopy from $\sigma_{c} \circ i_{c}$ to the identity, and thus that both $i_{c}$ and $\sigma_{c}$ induce isomorphisms on cohomology. This concludes the second proof. 
(3) As an application, suppose that $G$ is an analytic group over the field $k=\mathbb{Q}_{p}$. If $M$ and $N$ are two $k$-analytic manifolds, define $\mathscr{H} o m^{\text {an }}(M, N)$ as the set of $k$-analytic maps from $M$ to $N$. If $A$ is a finite-dimensional vector space over $k$ on which $G$ acts analytically, define $C_{\mathrm{an}}^{n}(G, A)$ to be $\mathscr{H}_{\mathrm{am}^{2 \mathrm{n}}}\left(G^{n+1}, A\right)$, on which $G$ acts in the obvious way. The cohomology of the complex $C_{\mathrm{an}}^{n}(G, A)^{G}$ is what Serre [7] calls the analytic cohomology $H_{\mathrm{an}}^{*}(G, A)$. If $H$ is a closed (hence by [2], $\S 8$. No. 2, $k$-analytic) subgroup of $G$ and $A$ is a finite-dimensional space over $k$ on which $H$ acts analytically, define $I_{A}^{\text {an }}=\operatorname{Ind}^{\text {an }}(A \mid H, G)$ to be the space of $k$-analytic maps $f: G \rightarrow A$ such that $f(g h)=h^{-1} f(g)$ for all $h \in H, g \in G$. Define the cohomology $H_{\mathrm{an}}^{*}\left(G, I_{A}^{\mathrm{an}}\right)$ by means of the cochain spaces $C_{\mathrm{an}}^{n}\left(G, I_{A}^{\mathrm{an}}\right)=$ $\mathscr{H} \operatorname{sm}_{H}^{\text {an }}\left(G^{n+1} \times G, A\right)$ (where $H$ acts on the final $G$ factor on the right). Because an analytic cross-section exists, the proof we have given in Remark (2) will work with only slight modifications in this situation as well to show that $H_{\mathrm{an}}^{*}\left(G, I_{A}^{\mathrm{an}}\right) \cong H_{\mathrm{an}}^{*}(H, A)$.

(4) It will be useful later on to have a proof of Proposition 1 more detailed than the one we have given.

The basic construction underlying the proof is this: By Lemma 1, we know that $C^{0}(X) \cong \operatorname{Ind}(X \mid 1, G)$ for any $G$-module $X$, and from this and Lemma 2 one can deduce that there is a natural map from $\operatorname{Hom}\left(C_{1}, C_{2}\right)$ to $\operatorname{Hom}_{G}\left(C^{0}\left(C_{1}\right), C^{0}\left(C_{2}\right)\right)$, for any two $G$-spaces $C_{1}$ and $C_{2}$. Explicitly, suppose that $\psi$ is a map from $C_{1}$ to $C_{2}$ (not necessarily a $G$-map). Then the map $\psi_{0}$ from $C^{0}\left(C_{1}\right)$ to $C^{0}\left(C_{2}\right)$ is that defined by the formula $\psi_{0}(\phi)(g)=g \psi\left(g^{-1} \phi(g)\right)$. If $\psi$ is itself a $G$-map, then of course $\psi_{0}(\phi)(g)$ is just $\psi(\phi(g))$, and this diagram commutes:

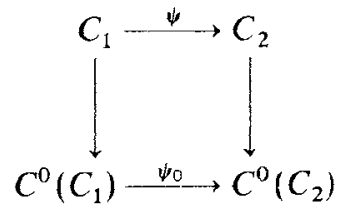

where the vertical arrows are the canonical injections. Since $C^{n}(X)=$ $C^{0}\left(C^{n-1}(X)\right)$, one can extend this construction to give maps

$$
\psi_{n}: C^{n}\left(C_{1}\right) \rightarrow C^{n}\left(C_{2}\right)
$$

Explicitly, $\psi_{n}(\phi)\left(g_{0}, g_{1}, \ldots, g_{n}\right)=g_{n} \psi\left(g_{n}^{-1} \phi\left(g_{0}, \ldots, g_{n}\right)\right)$.

Now let

$$
0 \longrightarrow A \stackrel{d_{-1}}{\longrightarrow} A^{0} \stackrel{d_{0}}{\longrightarrow} A^{1}-\stackrel{d_{1}}{\longrightarrow} \cdots
$$

be given, and let $\delta_{i}: A^{i} \rightarrow A^{i-1}$ be maps such that $I=d_{i-1} \delta_{i}+\delta_{i+1} d_{i}$ (which exist because $A^{*}$ is a strong resolution of $A$ ). From what we have said, there exist maps $d_{i}^{n}: C^{n}\left(A^{i}\right) \rightarrow C^{n}\left(A^{i+1}\right)$ and $\delta_{i}^{n}: C^{n}\left(A^{i}\right) \rightarrow C^{n}\left(A^{i-1}\right)$ such 
that $I=d_{i-1} \delta_{i}+\delta_{i+1} d_{i}$ and the following diagram commutes:

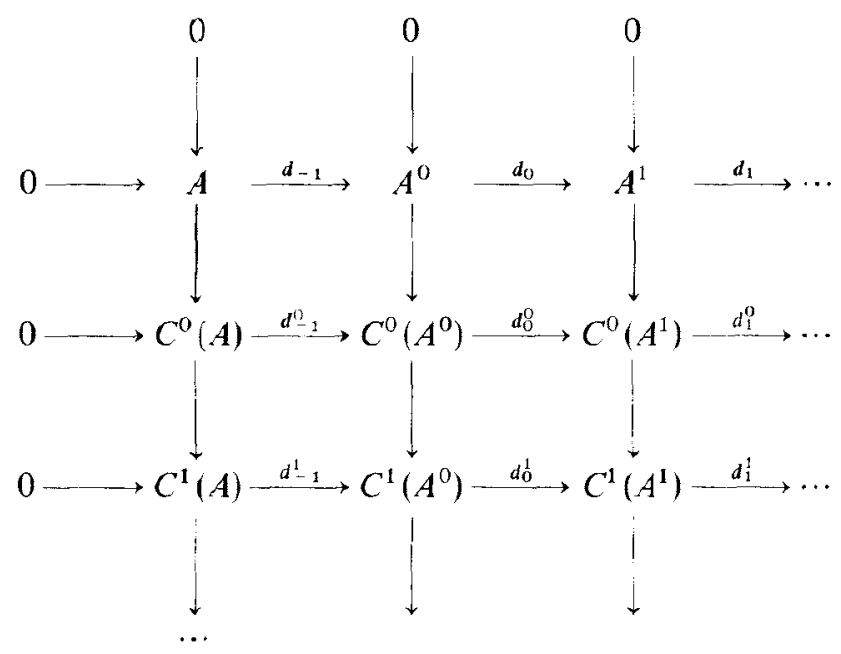

Furthermore, for $n \geqq 0$ all the maps $d_{i}^{n}$ and $\delta_{i}^{n}$ are $G$-maps. It is this which guarantees that each row

$$
0 \rightarrow C^{n}(A)^{G} \rightarrow C^{n}\left(A^{0}\right)^{G} \rightarrow C^{n}\left(A^{1}\right)^{G} \rightarrow \cdots
$$

is exact, and insures that one of the two spectral sequences we get collapses, proving the lemma.

2. Throughout this section, let $G$ be a locally compact group and $H$ a closed, normal subgroup.

Proposition 5. Let $A$ be a $G$-module such that (a) the groups $C^{n}(G, A)$ are continuously injective $H$-modules and (b) the cohomology $H^{*}(H, A)$ is strongly Hausdorff. Then the cohomology $H^{*}(H, A)$ has a canonical structure as a $G / H$-module, and there exists a spectral sequence converging to $H^{*}(G, A)$ with $E_{2}^{p, q} \cong H^{p}\left(G / H, H^{q}(H, A)\right)$.

Proof. The groups in the complex

$$
0 \rightarrow C^{0}(G, A)^{H} \rightarrow C^{1}(G, A)^{H} \rightarrow \cdots
$$

are continuously injective $G / H$-modules. Condition (a) implies that the cohomology of this complex is $H^{*}(H, A)$. Apply Proposition 2 to finish the proof.

Remarks. (1) Calvin Moore has for his cohomology a stronger result ([10], §9) assuming only that $H^{*}(H, A)$ is Hausdorff, which is the weakest natural assumption in view of the fact that it should be a module for $G / H$. 
(2) The spectral sequence always exists when $H$ is open in $G$, hence $G / H$ discrete. (This case was dealt with by Lazard in [7].) This covers one of the two cases we shall apply it to in this paper; the other is covered by the even easier case when $A$ is a vector space over a field of characteristic 0 , and $H$ is finite, so that the complex in the proof is exact.

Now let $\mathbb{F}$ be any field with a valuation inducing a non-trivial topology, complete with respect to this valuation. A number of useful cases are covered by:

Proposition 6. Suppose $H$ is $\sigma$-compact, A any complete metrizable topological vector space over $\mathbb{F}$. If $H^{n}(H, A)$ has finite dimension over $\mathbb{F}$, then it is strongly Hausdorff.

Proof. The spaces $C^{n}(H, A), Z^{n}=$ cocycles in $C^{n}$, and $B^{n}=C^{n-1} / Z^{n-1}$, are all complete and metrizable over $\mathbb{F}$. Let $l$ be the canonical injection of $B^{n}$ into $Z^{n}$. Let $W$ be some complement (finite-dimensional by assumption) to $l\left(B^{n}\right)$. The obvious map from $B^{n} \times W$ to $Z^{n}$ is a bijection, hence an isomorphism by Corollary 1 to Theorem 1 of Chapter I, $\S 3$ in [3].

3. Let $k$ be $\mathbb{Q}_{p}$. Assume $\mathbb{G}$ to be a connected semi-simple algebraic group defined over $k$, and $G$ the group of its $k$-rational points. Let $\mathscr{G}$ be the Lie algebra of $G$.

Theorem 1. If $A$ is a finite-dimensional vector space over $k$ on which $G$ has an algebraic representation, then $H^{*}(G, A) \cong H^{*}(\mathscr{G}, A)$.

(The cohomology of $\mathscr{G}$ is the continuous cohomology. That of $\mathscr{G}$ is the usual Lie algebra cohomology. It is immediate that the representation of $G$ induces one of $\mathscr{G}$ since it corresponds to an algebraic homomorphism from $G$ to $G L(A)$.)

Proof. In several steps.

Step (1). First assume $\mathbb{G}$ is simple, simply-connected, and isotropic. One has associated to $G$ its Bruhat-Tits building $\mathscr{B}$, a complex whose simplices are parametrized by certain open compact subgroups of $G$, the parahoric subgroups (we refer to [8] for details). Choose $l+1$ fixed maximal compacts $K_{1}, K_{2}, \ldots, K_{l+1}$ containing a fixed Iwahori subgroup. Then the parahorics containing this lwahori subgroup are in one-one correspondence with subsets $\alpha \subseteq t=\{1,2, \ldots, l+1\}$, the subset $\alpha$ corresponding to $\bigcap_{i \in \alpha} K_{i}$ (so that the Iwahori subgroup itself is $K_{t}$ ). Every parahoric subgroup of $G$ is conjugate to some $K_{\alpha}$. If $\sigma$ is the simplex of $\mathscr{B}$ associated to a parahoric $K$ which is conjugate to $K_{\alpha}$, we say $\sigma$ is of type $\alpha$. Its dimension will be $\operatorname{card}(\alpha)-1$.

The group $G$ acts on $\mathscr{B}$, transitively on the simplices of dimension $l$. The simplices of type $\alpha$ are isomorphic to $G / K_{\alpha}$ as a $G$-set. 
For each $\alpha \subseteq l$, let $\sigma_{\alpha}$ be the simplex associated to $K_{\alpha}$.

The $q$-simplices in $\mathscr{B}$ form a $G$-space $\Sigma_{q}$, with the discrete topology. If $M$ is a $G$-module, define the module $B^{q}(M)$ of $q$-cochains on $\mathscr{B}$ with values in $M$ to be $\operatorname{Hom}\left(\Sigma_{q}, M\right)$. By Lemma 1 ,

$$
B^{q}(M) \cong \sum_{\operatorname{card}(\alpha)=q+1} \operatorname{Ind}\left(M \mid K_{\alpha}, G\right)
$$

as a $G$-module. By Proposition 3, therefore, since $G / K_{\alpha}$ is discrete, one has

$$
H^{*}\left(G, B^{q}(A)\right) \cong \sum_{\operatorname{card}(\alpha)=q+1} H^{*}\left(K_{x}, A\right) .
$$

Further, since $\mathscr{B}$ is contractible (see [5], Appendix 2), for any $G$-module $M$ the cochain complex

$$
0 \rightarrow M \rightarrow B^{0}(M) \rightarrow B^{1}(M) \rightarrow \cdots \rightarrow B^{l}(M) \rightarrow 0
$$

is a strong resolution of $M$. Thus, one may apply Eq. (1) and Proposition 1 to deduce that there is a spectral sequence converging to $H^{*}(G, M)$ whose $E_{2}^{p, q}$-term is the $q$-th cohomology of the complex

$$
0 \rightarrow \sum_{\operatorname{card}(\alpha)=1} H^{p}\left(K_{\alpha}, M\right) \rightarrow \sum_{\operatorname{card}(\alpha)=2} H^{p}\left(K_{\alpha}, M\right) \rightarrow \cdots \rightarrow H^{p}\left(K_{\imath}, M\right) \rightarrow 0
$$

where all the maps are derived from the inclusions of $K_{\beta}$ in $K_{\alpha}$ when $\alpha \subseteq \beta$.

Now the groups $K_{\alpha}$ are compact, and to these one may apply Theorem 2.4.10 of Chapter $V$ in [7]. The group $H^{p}\left(K_{\alpha}, A\right)$ is therefore naturally isomorphic to $H^{p}(\mathscr{G}, A)^{K_{\alpha}}$. But one knows ([7] again) that the stabilizer of $H^{p}(\mathscr{G}, A)$ in $G$ is open, at least. Since the actions of $G$ on $\mathscr{G}$ and $A$ are algebraic, so is that on $H^{*}(\mathscr{G}, A)$. Therefore, the stabilizer is Zariskiopen, therefore all of $G$. Thus each $H^{p}\left(K_{\alpha}, A\right)$ is in fact just $H^{p}(\mathscr{G}, A)$, and the complex above is just that of the simplex $\sigma_{i}$, with coefficients in $H^{p}(\mathscr{G}, A)$. Hence only the 0 -th cohomology of this complex is $\neq 0$, and from this the theorem follows in this case.

Step (2). Next assume $\mathbb{G} \cong \Pi \mathbb{G}_{i}$, with $G_{0}$ anisotropic and each $\mathbb{G}_{i}(i \geqq 1)$ simple, simply connected, and isotropic. The same argument as above works, essentially, since $G$ acts on the product of the Bruhat-Tits buildings $\mathscr{B}_{i}(i \geqq 1)$.

Step (3). Allow $\mathbb{G}$ to be general, $\tilde{\mathbb{G}}$ its simply connected covering, $\tilde{G}$ the corresponding group of $k$-rational points, $\varphi: \tilde{G} \rightarrow G$ the canonical map. One knows that $\operatorname{Ker}(\varphi)$ is finite and that $\varphi(\tilde{G})$ is normal and has finite index in $G$. Let $\tilde{G}$ act on $A$ through $\varphi$. Applying Hochschild-Serre to $(\operatorname{ker} \varphi, \tilde{G})$, one has that $H^{*}(\varphi(\tilde{G}), A) \cong H^{*}(\tilde{G}, A)$ which in turn is isomorphic to $H^{*}(\mathscr{G}, A)$ by Step (2). Applying it to $(\varphi(\tilde{G}), G)$ one has that $H^{*}(G, A) \cong H^{*}(\varphi(\tilde{G}), A)^{G} \cong H^{*}(\mathscr{G}, A)^{G}$. Since $\varphi(\tilde{G})$ acts trivially on 
$H^{*}(\mathscr{G}, A)$ and its Zariski closure is all of $G$, this last is just $H^{*}(\mathscr{G}, A)$, and the theorem is proven.

This proof is the $p$-adic analogue of the proof of Theorem 6.1 in [4].

4. Assume $\mathbb{G}$ to be as in $\S 3$. We now discuss modules which are quasi-complete locally convex topological vector spaces over $\mathbb{R}$.

Lemma 7. If $K$ is any compact topological group and $V$ a quasicomplete locally convex topological vector space over $\mathbb{R}$, then $H^{n}(K, V)=0$ for $n>0$.

Proof. If $V$ is quasi-complete, so is $C^{n}(K, V)$, for each $n$. Therefore, it is sufficient to show that any strong short exact sequence of quasicomplete, etc., $K$-spaces splits with respect to $K$. But if

$$
0 \rightarrow U \rightarrow V \rightarrow W \rightarrow 0
$$

is such a sequence and $\sigma: W \rightarrow V$ is an $\mathbb{R}$-splitting then the map $\sigma_{K}: w \rightarrow \int_{K} k \cdot \sigma\left(k^{-1} w\right) d k$ (assuming $K$ to have total measure 1) is a continuous $K$-splitting.

Assume that $\mathbb{G}$ is simple, simply connected, and isotropic. We follow the notation used in Step (1) of the proof of Theorem 1. Let $A$ be a quasicomplete, etc., space.

Theorem 2. The cohomology $H^{*}(G, A)$ is that of the complex

$$
0 \rightarrow \sum_{\operatorname{card}(\boldsymbol{\alpha})=1} A^{K_{\alpha}} \rightarrow \sum_{\operatorname{card}(\alpha)=2} A^{K_{\alpha}} \rightarrow \cdots \rightarrow A^{K_{\mathrm{r}}} \rightarrow 0 .
$$

Proof. As before, we have the strong resolution of $A$ :

$$
0 \rightarrow A \rightarrow B^{0}(A) \rightarrow \cdots \rightarrow B^{l}(A) \rightarrow 0 .
$$

We claim that $B^{q}(A)$ is now an acyclic $G$-module.

Proof of the claim: Use Lemma 7 and Eq. (1) in $\$ 2$.

By Proposition 1, the cohomology of $G$ is that of the complex

$$
0 \rightarrow B^{0}(A)^{G} \rightarrow B^{1}(A)^{G} \rightarrow \cdots \rightarrow B^{l}(A)^{G} \rightarrow 0 .
$$

Apply Lemma 3 to get the theorem.

Corollary 1. The module $A$ has trivial cohomology unless $A^{K_{z}} \neq 0$.

Corollary 2. One has $H^{n}(G, \mathbb{C})=0$ for $n \neq 0$.

Proof. In this case, the cohomology of the complex in Theorem 2 is that of an $(l+1)$-dimensional simplex.

Define $A_{f}$ to be the subspace of all $a \in A$ such that $a \in A^{K}$ for some open subgroup $K$. If one gives $A_{f}$ the trivial topology, with respect to 
which every linear functional on $A$ is continuous, then $A_{f}$ is again quasicomplete, etc., and $G$ acts continuously on it.

Corollary 3. The inclusion of $A_{f}$ into $A$ induces an isomorphism $H^{*}\left(G, A_{f}\right) \cong H^{*}(G, A)$.

Proof. The complex in Theorem 2 is the same for $A_{f}$ and $A$.

Remarks. (1) Let $\mathbb{F}$ be any field of characteristic 0 , and let $V$ be a vector space over $\mathbb{F}$, with the discrete topology. A smooth representation of any locally compact totally disconnected group on $V$ is one which is continuous with respect to this topology (see [6]). If $K$ is a compact totally disconnected group with a smooth representation on $V$, then each $C^{n}(K, V)$ is again smooth. Lemma 7 holds in this situation, since the integrals involved are merely sums (the observation that this can be done for arbitrary characteristic 0 fields was made by Borel), and the analogue of Theorem 2, with $A$ a smooth representation of $G$, may be proved exactly as Theorem 2 itself.

(2) One may take the field $k$ in this section (the field of definition of $\mathbb{G}$ ) to be any locally compact non-archimedean field.

5. Again assume $\mathbb{G}$, etc., as in $\S 3$. The following answers another question of Serre's in [11] (see Remark (3) in $\S 1$ for the definition of $H_{a n}^{*}$ ):

Theorem 3. If $A$ is a vector space of finite dimension over $k$ on which $G$ has an algebraic representation, then $H_{\mathrm{an}}^{*}(G, A)=H^{*}(\mathscr{G}, A)$.

Proof. Lazard has shown ([7], Chapter V, Theorems 2.3 .10 and 2.4 .10 ) that if $K$ is a compact $k$-analytic group acting continuously (hence analytically by [2], $\S 8$, Theorem 1) on $A$, then $H_{\text {an }}^{*}(K, A) \cong H^{*}(k, A)^{K}$, where $k$ is the Lie algebra of $K$. Therefore, the proof of Theorem 3 proceeds as the proof of Theorem 1 does, once one has established the existence of a spectral sequence which converges to $H_{\mathrm{an}}^{*}(G, A)$ and has as $E_{2}^{p, q}$ term the $q$-th cohomology of the complex

$$
0 \rightarrow \sum_{\operatorname{card}(\alpha)=1} H_{\mathrm{an}}^{p}\left(K_{\alpha}, A\right) \rightarrow \cdots \rightarrow H_{\mathrm{an}}^{p}\left(K_{\imath}, A\right) \rightarrow 0 .
$$

To get the analogous result for the continuous cohomology, we applied Proposition 1; if one carefully analyzes its proof and refers to Remark (3) of $\S 1$, one sees that everything carries through if the following sequence is exact, for each $n \geqq 0$ :

$$
0 \rightarrow C_{\mathrm{an}}^{n}(A)^{G} \rightarrow C_{\mathrm{an}}^{n}\left(B^{0}(A)\right)^{G} \rightarrow \cdots \rightarrow C_{\mathrm{an}}^{n}\left(B^{l}(A)\right)^{G} \rightarrow 0 .
$$

Here, $C_{\text {an }}^{n}\left(B^{q}(A)\right)$ is defined implicitly in Remark (2) of $\S 1$, since

$$
B^{q}(A) \cong \sum_{\operatorname{card}(\alpha)=q+1} \operatorname{Ind}\left(A \mid K_{\alpha}, G\right)
$$


More explicitly, it consists of the maps $f$ from $G^{n+1}$ to $B^{q}(A)$ such that if $\sigma$ is any $q$-simplex of $\mathscr{B}$, then the function $f\left(g_{0}, g_{1}, \ldots, g_{n}\right)(\sigma)$ is $k$-analytic on $G^{n+1}$. In Remark (4) of $\S 1$, however, we showed how explicit homotopy maps $\delta_{*}$ on $B^{*}(A)$ give rise to $G$-maps $\delta_{i}^{n}: C^{n}\left(B^{i}(A)\right) \rightarrow C^{n}\left(B^{i-1}(A)\right)$ and one can see without much difficulty that $\delta_{i}^{n}$ takes $C_{\text {an }}^{n}\left(B^{i}(A)\right)$ to $C_{\mathrm{an}}^{n}\left(B^{i-1}(A)\right)$. This proves the theorem.

\section{References}

1. Bourbaki, N.: Topologie générale, Ch. X (2d edition). Paris: Hermann 1961

2. Bourbaki, N.: Groupes et algèbres de Lie, Ch. III. Paris: Hermann 1972

3. Bourbaki, N.: Espaces vectoriels topologiques, Ch. I and II (2d edition). Paris: Hermann 1966

4. Hochschild, G. P., Mostow, G. D.: Cohomology of Lie groups. Illinois Jour. of Math. 6, 367-401 (1962)

5. Garland, H.: p-adic curvature and the cohomology of discrete subgroups of $p$-adic groups. Annals of Math. 97, 375-423 (1973)

6. Harish-Chandra: Harmonic analysis on reductive p-adic groups. Proc. Sympos. Pure Math. 25, Amer. Math. Soc., Procidence, 1973

7. Lazard, M.: Groupes analytiques $p$-adiques. Publ. Math. I.H.E.S. No. 26, 1965

8. MacDonald, I. G.: Spherical functions on a group of $p$-adic type. Ramanujan Institute, Madras, 1971

9. Michael, E.: Selected selection theorems. Amer. Math. Monthly. 63, 233-238 (1956)

10. Moore, C.: Group extensions and group cohomology. In the Batelle Seattle Summer Rencontres. Lecture Notes in Physics 6. New York: Springer 1970

11. Serre, J.-P.: Cohomologie des groupes discrets. In: Prospects of Mathematics, Ann. of Math. Studies 70, Princeton, 77-169 (1971)

12. Weiss, E.: Cohomology of Groups. New York: Academic Press 1969

13. Wigner, D.: Algebraic cohomology of topological groups. Trans. Amer. Math. Soc. 178, 83-93 (1973)

W. Casseiman

Department of Mathematics

University of British Columbia

Vancouver 8, Canada
D. Wigner

Department of Mathematics

University of Michigan

Ann Arbor, Michigan 48104, USA 